Investigación

\title{
CALIDAD DE VIDA EN PERSONAS MAYORES DE 60 AÑOS CON DIAGNÓSTICO DE ENFERMEDAD DE ALZHEIMER, DEL POLICLÍNICO "PUENTES GRANDES", CUBA
}

\section{QUALITY OF LIFE IN PEOPLE OVER 60 WITH A DIAGNOSIS OF} ALZHEIMER DISEASE, FROM THE "PUENTES GRANDES" POLYCLINIC, CUBA

\section{Victoria de la Caridad Ribot Reyes}

Médico Especialista de II grado en Medicina General Integral y en Psiquiatría, Máster en Longevidad Satisfactoria, Síndromes Demenciales y en Bioética, Profesora e Investigadora Auxiliar. Psiquiatra forense del Instituto de Medicina Legal. Ave. 21 no. 5005 apto. 5 e/50 y 52 cp. 11300. La Habana, Cuba

\section{Antonio Lázaro González Castillo}

Enfermero Máster en Enfermería, Profesor Asistente. Jefe de la sala de Psiquiatría del Hospital Docente Clínico Quirúrgico “Joaquín Albarrán”. La Habana, Cuba

\section{Niurka Chang Paredes}

Médico Especialista de I grado en Medicina General Integral y en Psiquiatría. Psiquiatra del policlínico "Puentes Grandes". La Habana, Cuba

Artículo recibido el 27 de junio de 2018. Aceptado en versión corregida el 29 de noviembre de 2018 .

\section{RESUMEN}

Se realizó un estudio descriptivo de corte trasversal con el objetivo de determinar cómo evalúan su calidad de vida de las personas mayores de 60 años con diagnóstico de demencia por Enfermedad de Alzheimer. Participaron 35 personas. Se estudiaron características sociodemográficas, relacionadas con la propia enfermedad y grado de dependencia. Para evaluar calidad de vida se utilizó el instrumento Calidad de vida en la Enfermedad de Alzheimer (ADRQL) y para el grado de dependencia el Índice de Barthel. Predominó el sexo femenino (60\%), las edades entre los 70 y 79 años (37.1\%), para una edad media de 78,1 años con desviación estándar de $\pm 11,2$, las personas viudas $(42,9 \%)$ y divorciadas $(31,4 \%)$, con nivel de escolarización primario (40\%), la enfermedad demencial leve (60\%) y la dependencia grave $(37,1 \%)$ y leve $(31,4 \%)$. La mayoría de las personas estudiadas evaluó su calidad de vida global como satisfactoria $(65,7 \%)$. Los mejores resultados se obtuvieron en el área identidad personal y de personas importantes (74,3\%). El área comportamiento en el lugar de residencia recibió más valoraciones negativas $(57,1 \%)$.

Palabras clave: Calidad de vida, demencia, enfermedad de Alzheimer, cuidadores. 


\begin{abstract}
A descriptive cross-sectional study was carried out in order to determine how they assess their quality of life for people over 60 years of age with a diagnosis of dementia due to Alzheimer's disease. 35 people participated. Sociodemographic characteristics related to the disease itself and degree of dependence were studied. To assess the quality of life, the instrument Quality of life in Alzheimer's disease (ADRQL) was used, and for the degree of dependence, the Barthel Index. Female sex predominated (60\%), ages between 70 and 79 years $(37.1 \%)$, for an average age of 78.1 years with standard deviation of \pm 11.2 , widowed $(42.9 \%)$ and divorced $(31.4 \%)$, with primary schooling level $(40 \%)$, mild dementia (60\%) and severe $(37.1 \%)$ and mild $(31.4 \%)$. The majority of the people studied evaluated their overall quality of life as satisfactory $(65.7 \%)$. The best results were obtained in the area of personal identity and of important people (74.3\%). The behavior area in the place of residence received more negative evaluations $(57.1 \%)$.
\end{abstract}

Key words: Quality of life, dementia, Alzheimer's disease, caregivers.

\title{
http://dx.doi.org/10.7764/Horiz_Enferm.29.3.180-191
}

\section{INTRODUCIÓN}

A pesar de la gran atención que ha recibido el concepto calidad de vida $(\mathrm{CV})$, todavía no se ha logrado una definición consensuada del mismo dada su naturaleza multidimensional, dinámica y la diversidad de variables por las que se ve influida $^{1,2}$. Entre las más completa está la que ofrece la Organización Mundial de la Salud (OMS): la manera en que el individuo percibe su vida, el lugar que ocupa en el contexto cultural y el sistema de valores en que vive, la relación con sus objetivos, expectativas, normas, criterios y preocupaciones, todo ello permeado por las actividades diarias, la salud física, el estado psicológico, el grado de independencia, las relaciones sociales, los factores ambientales $y$ sus creencias personales $^{3}$.

Anualmente se reportan 4, 6 millones de casos nuevos de demencia a nivel mundial; ${ }^{4}$ por lo que, la promoción del bienestar y el mantenimiento de una buena $\mathrm{CV}$ resultan fundamentales para las personas que la padecen, debido a la dependencia que entidad genera. Los ancianos con diagnóstico de demencia no sólo se enfrentan a las pérdidas relacionadas con la enfermedad, sino también a las experiencias que acompañan a la vejez, como son el deterioro, la soledad y el aislamiento social ${ }^{5}$.

Con el nombre de demencia se denomina un síndrome complejo de naturaleza orgánica, adquirido, caracterizado por la presencia de un deterioro permanente de la memoria y otras funciones intelectuales, frecuentemente acompañado de otras manifestaciones psicopatológicas y alteraciones del comportamiento, que ocurre sin afectación del nivel de conciencia ni depresión, y afecta al funcionamiento laboral y/o social del 
Ribot V., González, A., Chang, N.

sujeto. Se considera un síndrome clínico de etiología múltiple, por lo general de curso crónico y progresivo, pero no necesariamente irreversible ${ }^{6}$. Esta declinación progresiva de las funciones mentales es producida por diversos tipos de lesiones orgánicas del cerebro con suficiente severidad para que afecte el normal desenvolvimiento del paciente en la sociedad o en la familia ${ }^{7}$.

Las entidades que pueden dar lugar a una demencia son muy numerosas. Suelen considerarse 3 grandes grupos etiológicos: demencias degenerativas primarias (p. ej., enfermedad de Alzheimer); demencias secundarias (p. ej., demencia vascular) y demencias combinadas o de múltiple etiología (p. ej., demencia mixta o demencia tipo Alzheimer con enfermedad cerebrovascular asociada). La forma de demencia más común es la enfermedad de Alzheimer (60\%), seguida de la demencia vascular (15-20\%) y la demencia asociada a la enfermedad de Parkinson (10-15\%). Estas formas más frecuentes de demencia son irreversibles, pero hay que tener en cuenta que un 5-10\% de las demencias son potencialmente reversibles, y entre ellas hay que destacar las asociadas al empleo de fármacos ${ }^{6}$.

La enfermedad de Alzheimer, es el tipo de Demencia más frecuente, afecta alrededor de un $6 \%$ de las personas mayores de 65 años, su incidencia se incrementa con la edad, calculándose que está presente en el $20 \%$ de las personas de 80 y más años.

En el contexto cubano, el envejecimiento poblacional es ya una realidad declarada, dato que corroboran los censos ${ }^{8}$ realizados en los últimos años y el Anuario estadístico de salud $2015^{9}$. De acuerdo a estos, el país ha transitado desde un $11,3 \%$ personas de 60 años y más en 1985, a 18, 3\% en el año 2012 y 19, 4\% en el 2015; cifra que apunta a 2.158 .703 de personas mayores de 60 años. Por lo que representa un aumento del envejecimiento poblacional en 8,1 puntos porcentuales en un período de solo 30 años. Tales estimaciones señalan a la menor de las Antillas como uno de los países más envejecidos de la región en el $2050^{10}$.

La prevalencia del síndrome demencial oscila entre 6,4 y 10,2 en la población de 65 y más años ${ }^{11}$ y se espera que número de cubanos con demencia se incremente en 2,3 veces para el año $2040^{7}$.

En las personas con demencia, la $\mathrm{CV}$ es una de las variables más importantes para estudiar la eficacia de intervenciones. Sin embargo, su evaluación es difícil porque:

a) este es un constructo complejo para el que no existe una aproximación teórica o conceptual unificada.

b) al tratarse de personas con deterioro cognitivo se complica enormemente la obtención de información fiable ${ }^{12}$.

Aun así, la medición de la CV es imprescindible en la valoración de la eficacia de cualquier intervención terapéutica y en la organización de la actuación asistencial. Además, la evaluación seriada de la CV durante el curso de la enfermedad puede aportar datos valiosos acerca de los aspectos más relevantes que tratar en cada estadio ${ }^{13}$.

En Cuba, no se recogen estudios que evalúen el comportamiento de esta variable en pacientes con Enfermedad de Alzheimer u otro tipo de demencia. Es por ello que, a partir lo anteriormente 
Calidad de vida en personas mayores de 60 años...

expuesto, nos motivamos a llevar a cabo un estudio con el objetivo de determinar cómo evalúan las personas mayores con diagnóstico de demencia que reciben atención en el policlínico "Puentes Grandes", su CV.

\section{METODOLOGÍA}

Se realizó un estudio descriptivo de corte transversal para determinar cómo evalúan su CV las personas mayores de 60 años con diagnóstico de demencia del policlínico "Puentes Grandes", en el periodo comprendido entre septiembre y noviembre del 2017. Se trabajó con una muestra no probabilística e intencional, formada por 35 personas. Se exploraron variables sociodemográficas, relacionadas con el síndrome demencial y grado de dependencia.

\section{Instrumento recolector de datos}

Para evaluar la CV se aplicó la escala CV en la Enfermedad de Alzheimer (ADRQL), creada por Rabins PV et al. ${ }^{14}$.

Se trata de un instrumento específicamente diseñado para evaluar la calidad de vida del paciente con demencia tipo Alzheimer. Es una escala heteroaplicada; el marco de referencia temporal son las dos semanas previas. Consta de 47 ítems y se divide en cinco dominios importantes: 1. Interacción social, 2. Disfrute de las actividades, 3. Sentimientos y conductas estresantes, 4. Humor y actividades cotidianas y 5 . Respuesta al entorno; los cuales se puntúan mediante una escala dicotómica de acuerdo/desacuerdo. La puntuación total para el ADRQL se calcula mediante la suma de los valores asignados a las respuestas, dividiendo la suma por el valor máximo de la escala y multiplicando los resultados por 100 para obtener un porcentaje de 0 a $100 \%$. Las puntuaciones se pueden calcular de la misma manera para los cinco dominios de la escala. A mayor puntuación en la escala, mejor será la CV del paciente, por encima del $70 \%$ en la evaluación se considera satisfactoria.

Para aplicarla en nuestro medio, Se utilizó la metodología propuesta por Moriyama $\mathrm{IM}^{15}$, para evaluar la calidad de su construcción y la correspondencia de los ítems con la definición operacional y las categorías que la componen. La estructura y formulación de las preguntas del cuestionario fue sometido a un proceso de validación de contenido por criterio de expertos. El criterio de los jueces expertos se utilizó para evaluar la correspondencia de los ítems en relación con la definición operacional y las categorías propuestas y para evaluar el cuestionario de acuerdo a las cinco propiedades básicas formuladas por Moriyama. La respuesta de los expertos, valoración que ellos realizaron sobre las referidas propiedades se recogió a través de una escala ordinal que incluyó: Mucho, Poco y Nada. El cuestionario fue sometido, antes de su aplicación con el objetivo de validación, a un proceso de pilotaje. Fue aplicado a cinco investigadores considerados como expertos, cuyas sugerencias permitieron su corrección quedando así confeccionado el cuestionario de validación definitivo. También se realizó se realizó un estudio piloto en un grupo de 11 pacientes, para comprobar la comprensión de las preguntas y el funcionamiento del instrumento.

La selección de los jueces expertos, se realizó teniendo en consideración su 
Ribot V., González, A., Chang, N.

experiencia profesional e investigativa en el ejercicio de las ciencias médicas y psicológicas, para de este modo tener la posibilidad de obtener criterios de diversos especialistas y realizar un análisis integral del problema de estudio. Se incluyeron profesionales considerados expertos en psicología, medicina interna, geriatría, psiquiatría y medicina general integral, que ejercieran su actividad profesional en los tres niveles de atención, en dos provincias del país. Se seleccionaron siete jueces expertos siguiendo los criterios mencionados anteriormente, quienes respondieron el cuestionario de validación definitivo. La validez de construcción del cuestionario fue evaluada a través de un análisis factorial por componentes principales utilizando el método de rotación Varimax. Se realizó un análisis de las comunalidades para conocer la representatividad de las variables en los factores retenidos y de la consistencia interna por factor retenido.

Para determinar el grado de dependencia se utilizó el Índice de Barthel. ${ }^{16}$ Instrumento heteroaplicado y validado en nuestro medio ${ }^{17}$. Evalúa 10 actividades básicas de la vida cotidiana: comer, lavarse, vestirse, arreglarse, deposiciones, micción, usar el retrete, trasladarse, deambular y escalones. El marco referencial es de hasta una semana previa.

Se vaciaron los datos obtenidos en una base de datos creada en el programa estadístico SPSS versión 11.5, se utilizó estadísticas descriptivas como las distribuciones de frecuencias, cálculos porcentuales. Posteriormente, se representaron en tablas con el uso de Microsoft Word.

Se solicitó autorización a la dirección y al comité científico del centro para llevar a cabo la investigación. A todos los participantes en el estudio se les ofreció una detallada información sobre los objetivos de la investigación y se les solicitó su consentimiento para participar en la misma. Se cumplieron todos los requisitos establecidos en la Declaración de Helsinki del $2013^{18}$.

\section{RESULTADOS}

Predominó el sexo masculino $(60,0 \%)$, los viudos(as) $(42,9 \%)$ y el nivel educacional primario $(40,0 \%)$ (Tabla 1$)$. La mayor parte de los participantes se encontraban entre los 70 y 89 años $(68,5$ $\%)$, con una edad media de 78,1 años y una desviación estándar de \pm 11.2 .

Las formas leves (60\%) y moderadas $(28,6 \%)$ fueron las encontradas con mayor frecuencia (Tabla 2).

El mayor número de pacientes se consideraron dependientes graves $(31,7 \%)$, seguidos por los dependientes leves $(31,4 \%)$. Se encontraron 7 personas con encamamiento prolongado, las cuales fueron valoradas como dependientes totales. El $60 \%$ de la muestra presentaba un cuadro demencial leve (Tabla 3). 
Ribot V., González, A., Chang, N.

Tabla 1. Distribución de pacientes de acuerdo a variables sociodemográficas.

\begin{tabular}{llcc}
\hline \multicolumn{2}{c}{ Variables Sociodemográficas $(\mathbf{N}=35)$} & $\mathbf{N}^{\circ}$ & $\mathbf{\%}$ \\
\hline Sexo & Femenino & 21 & 60,0 \\
& Masculino & 14 & 40,0 \\
& $60-69$ & 6 & 17,4 \\
& $70-79$ & 13 & 37,1 \\
& $80-89$ & 11 & 31,4 \\
Estado civil & $\geq 90$ & 5 & 14,3 \\
& Soltero(a) & & \\
& Casado(a) & 3 & 8,57 \\
& Divorciado(a) & 4 & 11,4 \\
& Viudo(a) & 11 & 31,4 \\
& Unión consensual & 15 & 42,9 \\
& & 2 & 5,71 \\
Nivel & Analfabeto & & \\
educacional & Primario & 1 & 2,9 \\
& Secundario & 14 & 40,0 \\
& Preuniversitario/Técnico medio & 5 & 34,3 \\
& Universitario & 3 & 8,57 \\
\hline
\end{tabular}

Fuente: base de datos

Tabla 2. Distribución de pacientes severidad del síndrome demencial.

\begin{tabular}{llcc}
\hline \multicolumn{2}{c}{ Variables Relacionadas con enfermedad demencial } & & \\
& $(\mathbf{N = 3 5})$ & $\mathbf{N}^{\circ}$ & $\mathbf{\%}$ \\
\hline Severidad del & Leve & 21 & 60,0 \\
Síndrome Demencial & Moderado & 10 & 28,6 \\
& Severo & 4 & 11,4 \\
\hline
\end{tabular}

Fuente: base de datos

Tabla 3. Distribución de pacientes según grado de dependencia.

\begin{tabular}{lcc}
\hline Grado de Dependencia & $\mathbf{N}^{\circ}$ & $\mathbf{\%}$ \\
\hline Totalmente dependiente & 7 & 20,0 \\
Dependiente grave & 13 & 37,1 \\
Dependiente moderado & 5 & 14,3 \\
Dependiente leve & 11 & 31,4 \\
Totalmente independiente & 9 & 25,7 \\
Total & 35 & 100 \\
\hline
\end{tabular}

Fuente: base de datos.
En cuanto a la $\mathrm{CV}$, fueron entrevistadas 24 personas con diagnóstico de demencia y 11 cuidadores (en los casos en que el grado de deterioro cognitivo impedía la aplicación del instrumento). De estos últimos, 8 eran mujeres $(72,7 \%)$, el rango de edades predominante osciló entre los 35 y 69 años (90,9\%). En la mayoría de los casos se trataba de hijo(a) s $(54,5 \%)$ y nueras/yernos $(27,3 \%)$. 
Ribot V., González, A., Chang, N.

El área en se obtuvieron mejores resultados en la escala, traducido en una CV más satisfactoria, fue la relacionada con la identidad personal y de personas importantes. En esta esfera se exploraron elementos tales como: conocer su rol dentro de la familia (abuelo(a), esposo(a), etc.), mantener comunicación sobre el trabajo previo y/o actividades diarias, dar opinión sobre las actividades domésticas rutinarias (menú, vestimenta, dónde sentarse, etc.), interés por los acontecimientos, lugares o hábitos de su pasado tales como viejos amigos, antiguas residencias, ir a la iglesia, rezar, etc. La única área en la que la calidad de vida fue valorada como no satisfactoria fue el comportamiento en el lugar de residencia. En la misma se exploró: disfrute de posesiones y pertenencias, preocupación por la seguridad de estas, satisfacción por permanecer en el lugar donde vive, salidas fuera del hogar y grado de confort dentro del mismo.

Al revisar la puntuación global, 23 personas $(65,7 \%)$ fueron consideradas como portadoras de una $\mathrm{CV}$ satisfactoria (puntuación por encima del 70\%) y 12 no satisfactoria $(34,3 \%)$. Cabe destacar que los peores resultados se correspondieron con las personas que mayor grado de dependencia presentaban y la entrevista fue realizada con los cuidadores (Tabla 4).

Resulta llamativo que el $65,7 \%$ de los pacientes demenciados evaluaron su calidad de vida como satisfactoria. Los peores resultados se correspondieron con las personas que mayor grado de dependencia presentaban y la entrevista fue realizada con los cuidadores.

Tabla 4. Distribución de pacientes según calidad de vida en las áreas estudiadas.

\begin{tabular}{|c|c|c|c|c|}
\hline \multirow[b]{2}{*}{ Áreas } & \multicolumn{2}{|c|}{$\begin{array}{c}\text { Calidad de Vida } \\
\text { Satisfactoria }\end{array}$} & \multicolumn{2}{|c|}{$\begin{array}{c}\text { Calidad de Vida No } \\
\text { Satisfactoria }\end{array}$} \\
\hline & $\mathbf{N}^{\circ}$ & $\%$ & $\mathbf{N}^{\circ}$ & $\%$ \\
\hline Relaciones con otras personas & 21 & 60,0 & 14 & 40,0 \\
\hline $\begin{array}{l}\text { Identidad personal y de personas } \\
\text { importantes }\end{array}$ & 26 & 74,3 & 9 & 25,7 \\
\hline Conductas estresantes & 19 & 54,3 & 16 & 45,7 \\
\hline Actividades cotidianas & 22 & 62,9 & 13 & 37,1 \\
\hline $\begin{array}{l}\text { Comportamiento en el lugar de } \\
\text { residencia }\end{array}$ & 15 & 42,9 & 20 & 57,1 \\
\hline Puntuación Global & 23 & 65,7 & 12 & 34,3 \\
\hline
\end{tabular}

Fuente: base de datos.

\section{DISCUSIÓN}

Al revisar las publicaciones nacionales, se encontraron sólo revisiones teóricas sobre el tema ${ }^{20}$. Canciano Chirino ${ }^{4}$ exploró en el $2014 \mathrm{CV}$ en pacientes con demencia vascular post-ictus isquémico pero, el instrumento utilizado para medirla era específico para ictus, no para síndrome demencial. Consideramos que utilizar por primera vez la ADRQL en nuestro medio, abre las puertas para los investigadores que se desarrollan en este campo.

En la literatura internacional, el uso de instrumentos específicos para determinar $\mathrm{CV}$ en personas demenciadas, 
Ribot V., González, A., Chang, N.

se emplea desde hace relativamente pocos años. Inouye ${ }^{20}$ utilizó la Escala de Evaluación de CV en la Enfermedad de Alzheimer en 53 personas con diagnóstico de dicha patología y sus cuidadores. Encontró que los pacientes valoraban de forma más positiva su calidad de vida que lo que reportaban los cuidadores. Gómez ${ }^{21}$ llevó a cabo en España una investigación similar. Utilizó la escala QoL-AD (Quality of Life of Alzheimer Disease) en una muestra de 40 diadas conformadas por pacientes con enfermedad de Alzheimer en estadio leve-moderado. Constató que los pacientes tenían una percepción de vida más positiva en comparación con lo que opinaban sus cuidadores. Sin embargo, tuvo en consideración la posibilidad de que, por el deterioro mismo de los pacientes, la información dada no fuera lo suficientemente valida y confiable.

También en España, León Salas et al. ${ }^{22}$, utilizó la ADRQL en 200 pacientes con enfermedad de Alzheimer, con el objetivo de comparar diferencias en cuanto a la $\mathrm{CV}$ de los que vivían en residencias sanitarias y los que lo hacían en sus casas. En Colombia, Rodríguez ${ }^{23}$, utilizó la ADRQL para evaluar cambios en la CV de pacientes con demencia tipo Alzheimer, a partir de la aplicación de un programa de un programa de hatha-yoga.

Grske $^{24}$ aplicó la ADRQL en enfermos de Alzheimer, en cinco hogares de ancianos alemanes. Coincidiendo con nuestro estudio, las enfermeras y cuidadores realizaron valoraciones más negativas de la $\mathrm{CV}$ de los pacientes que ellos mismos.

Crespo $^{25}$ estudió la CV en población con demencia e institucionalizada en varias ciudades españolas ha sido estudiada a partir de las opiniones de los pacientes, cuidadores familiares y personal profesional de la institución. Los resultados indican que las puntuaciones de CV expresadas por los pacientes fueron significativamente más elevadas que las de los informantes, así que se obtuvieron correlaciones significativas entre las puntuaciones de los informantes (familiares y profesionales), pero éstas no correlacionaron con las de los residentes. Un análisis comparativo de la $\mathrm{CV}$ en pacientes con demencia residentes en vivienda familiar y en institución, evaluada por el cuidador más cercano (familiar o cuidador profesional, respectivamente), reveló que la CV total y la de los subdominios fue más elevada cuando fue valorada por el familiar.

Se ha detectado que trastornos como la sobrecarga y la depresión influyen en la visión negativa que el cuidador tiene sobre la calidad de vida del anciano dependiente y la suya propia ${ }^{26}$. También se ha planteado que la propia enfermedad y su repercusión en las funciones psicológicas superiores, le restan objetividad a la evaluación que el adulto mayor realiza ${ }^{27,28}$. Sin embargo, en la investigación llevada a cabo por Chirmuley $^{29}$, ni el estatus socioeconómico ni la inteligencia emocional, influyeron de manera significativa en los cuidadores a la hora de evaluar la CV de los enfermos con Alzheimer.

Los resultados obtenidos en
nuestro estudio le confirieron al
comportamiento en el hogar, los puntajes
más pobres en la escala. Dentro de esta
esfera, los ítems que más valoraciones
negativas tuvieron fueron los relacionados
con el disfrute de las pertenencias y grado


Calidad de vida en personas mayores de 60 años...

de confort dentro del hogar. Estos hallazgos son similares a los reportados por Lyketsos $^{30}$, en una investigación longitudinal de 2 años que incluyó 47 personas en centros de cuidados a largo plazo. En nuestro caso, consideramos que esto pudiera estar influenciado por los cambios en la dinámica familiar que impone la presencia de una persona con demencia y las dificultades que se derivan de la convivencia de familias multigeneracionales, sin el espacio y las condiciones necesarios dentro de las viviendas la mayoría de las ocasiones.

En nuestro estudio, las puntuaciones más bajas fueron obtenidas en las personas con mayor grado de dependencia y severidad del cuadro demencial, resultado similar al reportado por Missotten ${ }^{31}$ en el 2008. Sin embargo, en una investigación longitudinal con 117 sujetos por el mismo autor, se encontró que el análisis de datos ADRQL no reveló modificaciones significativas de la $\mathrm{CV}$ durante el período de 2 años, a pesar de que los resultados de los instrumentos clínicos mostraron un deterioro significativo ${ }^{21}$.

A pesar del debate que se genera en torno al tema, existe consenso en la CV se refiere a la percepción subjetiva de la persona sobre su estado de salud y la capacidad que tiene para lograr mantener un nivel de funcionamiento global, que le permita realizar las actividades de su interés, tanto si se trata del enfermo o de la persona que se desempeña el rol de cuidador. Por tanto, en enfermedades que afectan el Sistema Nervioso Central, como es el caso de las demencias, resulta vital tomar en cuenta para la adecuada evaluación la percepción acerca de la calidad de vida del enfermo, así como la de sus allegados, como una medida para complementar y verificar la información obtenida directamente de este, en aquellos momentos en que la persona, carece de un juicio objetivo por su mismo estado cognitivo ${ }^{26-28}$.

\section{CONCLUSIONES}

Para evaluar la CV en la demencia resulta pertinente tomar en cuenta no sólo los criterios del propio paciente, sino también las del cuidador. En nuestro estudio, el $65,7 \%$ de los participantes fueron considerados como portadores de una CV satisfactoria (puntuación por encima del $70 \%$ ) y en el $34,3 \%$ resultó no satisfactoria. Los peores resultados se correspondieron con las personas que mayor grado de dependencia presentaban y la entrevista fue realizada con los cuidadores Esta variable resulta ideal para determinar la eficacia de las estrategias terapéuticas y de intervención.

\section{REFERENCIAS BIBLIOGRÁFICAS}

${ }^{1}$ Sánchez Rodríguez JL, Torrellas Morales C, Fernández Gómez MJ, Martín-Vallejo J. Influencia de la reserva cognitiva en la calidad de vida en sujetos con enfermedad de Alzheimer. Anal Psicol [internet]. 2013 Oct [citado 18 Jul 2015]; 29(3): [aprox. 12]. Disponible en: http://dx.doi.org/10.6018/ analesps.29.3.138201

2. Serdá Ferrer BC. Calidad de Vida y Gerontología: Descripción de una línea de investigación. Eur J investig health psycho educa. 2014; 4 (3):301-8.

${ }^{3}$ Cardona Arias JA, Higuita Gutiérrez LF. Aplicaciones de un instrumento diseñado por la OMS para la evaluación de la calidad de vida. Rev Cubana Salud Pública 
Ribot V., González, A., Chang, N.

[Internet]. 2014 Abr-Jun [citado 18 Jul 2015]; 40(2):[aprox. 10]. Disponible en: http://scielo.sld.cu/scielo.php? script $=$ sci_arttext\&pid=S0864346620140 00200003

4. Canciano Chirino E, Valdés Busquet S, Sosa Pérez S, León López L. Calidad de vida en los pacientes con demencia vascular post-ictus isquémico (estudio de cohortes). Rev Cubana Neurol Neurocir. 2014; 4(1):7-13.

5. Rodríguez-Blázquez C, Martín-García S, Frades -Payo B, París MS, MartínezLópez I, João Forjaz M. Calidad de vida y estado de salud en personas mayores de 60 años con demencia institucionalizadas. Rev Esp Salud Pública [internet]. 2015 Ene-Feb [citado 18 Jul 2015]; 89(1):[aprox. 12]. Disponible en: http://scielo.isciii.es/scielo.php?pid=S113 557272015000100006\&script=sci_arttext \&tlng= 3Des

6. Martín Carrasco M. Actualización en el manejo de las demencias en atención primaria. JANO. 2008 Oct.; (1714): 4754.

${ }^{7}$ Llibre Rodríguez JJ. Introducción al estudio de las Demencias y Enfermedad de Alzheimer. [Presentación en PowerPoint] En: Bibliografía para la Maestría en Síndromes Demenciales. [CD-ROM] Universidad de Ciencias Médicas de La Habana: Facultad "Finlay-Albarrán"; 2015.

8. Oficina Nacional de Estadística [Internet]. La Habana: Oficina Nacional de Estadísticas e Información (ONEI); 2012 [actualizado 9 Oct 2011; citado 5 Jun 2015]. Censo de Población y Viviendas; [aprox. 2 pantallas]. Disponible en: http://www.one.cu/censo2012.htm
9. Anuario Estadístico de Cuba 2015 [Internet]. La Habana: Biblioteca Virtual de Salud en Cuba; 2016 [actualizado 9 Dic 2015; citado 5 Jun 2016]. Comparaciones internacionales. Indicadores seleccionados por países; [aprox. 2 pantallas]. Disponible en:

http://files.sld.cu/dne/files/2016/04/Anuar io_2015_electronico-1.pdf

${ }^{10}$ Ramos Monteagudo AM. Envejecimiento de la población en Cuba: logros vs desafíos. Archivo Médico de Camaguiey [Internet]. 2016 [citado 12 sept 2016]; 20(4). Disponible en: http://www.revistaamc.sld.cu/index.php/a mc/article/view/4371/2476

${ }^{11}$ Llibre Rodríguez JJ. Atención a personas con demencia y enfermedad de Alzheimer. La Habana: Ecimed; 2013.

12. Crespo L, Hornillo Jerez C, Bernardo de Quirós Aragón M, Gómez Gutiérrez MM. La evaluación de la calidad de vida en personas con demencia. Revista Española de Geriatría y Gerontología. Nov-Dec 2011; 46(6):319-24.

${ }^{13}$ Gómez-Gallego M, Gómez-Anor J, AdoGarcía M, Gómez García J. Estudio del acuerdo entre las valoraciones sobre la calidad de vida de pacientes con la enfermedad de Alzheimer y sus cuidadores. Alzheimer. Real Invest Demenc. 2012; 51:22-27.

${ }^{14}$ Rabins PV, Kasper JD, Kleinman L, Black BS. Concepts and methods in the development of the ADRQL: an instrument for assessing health-related quality of life in persons with Alzheimer's disease. J. Ment. Health Aging. 1999; 5: 33-48.

${ }^{15 .}$ Moriyama IM. Indicador of social change. Problems in the measurents of helth status. New York: Rusell Sage Foundation;1968. 
Calidad de vida en personas mayores de 60 años...

${ }^{16 .}$ Mahoney FI, Barthel DW. Functional evaluation: The Barthel Index. Md State Med J. 1965; 14(2): 61-5.

17. Govantes BY, Bravo AT. Estado funcional en pacientes con ictus isquémico. Hospital "Julio Díaz González”. Rev Cub de Med Fis y Rehab [internet]. 2014 [citado 11 May 2018]; 6 (2).

Disponible

en:

http://www.medigraphic.com/cgibin/new/resumen.cgi?IDARTICULO=55 905

18.World Medical Association. World Medical Association Declaration of Helsinki Ethical Principles for Medical Research Involving Human Subjects. Clinical Review \& Education. Special Communication; 2013.

${ }^{19}$ Ribot Reyes VC, Leyva Villafaña Y, Moncada Menéndez C, Alfonso Sánchez. Calidad de vida y demencias. Archivo Médico de Camagüey [Internet]. 2016 [citado 18 Sept 2016]; 20(1). Disponible en:

http://www.revistaamc.sld.cu/index.php/a mc/article/view/4156/2242

${ }^{20}$ Inouye K, Silva Pedrazzani E, Iost Pavarini SC, 4 Yoshie Toyoda C. Calidad de vida del anciano con enfermedad de Alzheimer: estudio comparativo de los relatos del paciente y del cuidador. Rev Latino-am Enfermagem [Internet]. 2010 ene-feb [citado 13 Abr 2015]; 18(1): [aprox. 7 p.]. Disponible en: www.eerp.usp.br/rlae

${ }^{21 .}$ Gómez M, Gómez J, Ato M, Gómez J. Estudio del acuerdo entre las valoraciones sobre la calidad de vida de pacientes con la enfermedad de Alzheimer y sus cuidadores. Madrid: Editorial Glosa S.L.; 2012.
22.León-Salas B, Olazarán J, Cruz-Orduña I, Agüera-Ortiz L, Dobato JL, Valentí-Soler M, Muñiz R, González-Salvador MT, Martínez-Martín P. Quality of life (QoL) in community-dwelling and institutionalized Alzheimer's disease (AD) patients. Arch Gerontol Geriatr [internet]. 2013 Nov-Dic [citado 11 May 2018];57(3):257-62. Disponible en: https://www.sciencedirect.com/science/ar ticle/pii/S0167494313000733

${ }^{23}$ Rodríguez MC, Guzmán LA, Llanos OL, Reyes A. Estudio piloto: efectos de un programa de hatha-yoga sobre variables psicológicas, funcionales y físicas, en pacientes con demencia tipo alzheimer. Psychol. av. discip.[internet] 2011 Dic. [citado 11 May 2018]; 5(2). Disponible en: http://www.scielo.org.co/scielo.php?pid= S1900-

23862011000200004\&script=sci_arttext \&tlng=en

${ }^{24 .}$ Grske J, Meyer S, Wolf-Ostermann K. Quality of life ratings in dementia care? a cross-sectional study to identify factors associated with proxy-ratings. Health and Quality of Life Outcomes [internet]. 2014 [citado 11 May 2018]; 12(177). Disponible en: http://www.hqlo.com/content/12/1/177

${ }^{25 .}$ Crespo MM, Bernaldo de Quirós MM, Gómez M, Hornillos C. Quality of life of nursing home residents with dementia: A comparison of perspectives of residents, family, and staff. The Gerontologist. 2012; 52(1): 56-65.

26. Garzón-Maldonado FJ, Gutiérrez-Bedmar M, García-Casares M, Pérez-Errázquin F, Gallardo-Tur A, Martínez-Valle Torre MD. Calidad de vida relacionada con la salud en cuidadores de pacientes con enfermedad de Alzheimer. Neurología 
Ribot V., González, A., Chang, N.

[Internet]. 2016 [citado 3 Ene 2017]; $\mathrm{XXX}(\mathrm{XX})$ : aprox. 8 p.]. Disponible en: http://dx.doi.org/10.1016/j.nrl.2016.02.02 3

27. Osorio Montoya LA, Salgado Forero MM. Calidad de vida en pacientes con demencia tipo Alzheimer. [Tesis] Bogotá: Pontificia Universidad Javeriana, Facultad de Psicología; 2014.

28.Perales J, D Cosco T, Blossom S, Fleming J, Martin S, Haro JM, et. al. Health-related quality of life in the Cambridge City over75s Cohort (CC75C): development of a dementia-specific scale and descriptive analyses. BMC Geriatrics [internet]. 2014 [citado 11 Ene 2017]; 14(18). Disponible en: http://www.biomedcentral.com/14712318/14/18

29. Chirmuley A. The Relationship between Emotional Intelligence and Socioeconomic status of caregivers on the Quality of Life in patient's with Alzheimer's dementia Indian Journal of
Mental Health [internet]. 2015 [citado 11 May 2018]; 2(2). Disponible en: http://indianmentalhealth.com/images/201 5-vol2-issue2/OR2-ASIRA-

ALZHEIMERS-PAPER.pdf

${ }^{30}$. Lyketsos CG, Gonzales-Salvador T, Chin JJ, Baker A, Black B, Rabins P. A followup study of change in quality of life among persons with dementia residing in a longterm care facility. Int J Geriatr Psychiatry. 2003; 18: 275-81.

${ }^{31 .}$ Missotten P, Squelard G, Ylieff M, Di Notte D, Paquay L, De Lepeleire J, et. al. Relationship between Quality of Life and Cognitive Decline in Dementia. Dement Geriatr Cogn Disord [internet]. 2008 [citado 11 May 2018]; 25:564-72. Disponible en: https://doi.org/10.1159/000137689

${ }^{32}$ Missotten P, Ylieff M, Di Notte D, Paquay L, De Lepeleire J, Buntinx F, et al. Quality of life in dementia: a 2-year follow-up study. Int. J. Geriatr. Psychiatry. 2007; 22: 1201-7. 